few resources to support people once they have given up drugs.

Resource needs are modest as, with imagination, existing local services could be used-for example, links could be established between adult education, further education, and sports centres and Manpower Services Commission projects. Making links, however, needs sensitive handling as people do not wish to be identified as ex-drug users. Links also need to be flexible as people would enter courses at various intervals, which may not always be convenient for course organisers. In addition, drop out rates may be high, which might affect people's willingness to take ex-users into their schemes. Because of the difficulties of making links people may be tempted to set up special rehabilitation facilities for drug misusers alone, yet this has a disadvantage as grouping drug misusers together sets up yet another ghetto type of environment. Instead of being prepared for a new lifestyle, past misusers are kept in a drug culture environment, and their resolution to give up drugs may be destroyed by less motivated members of the group.

Medway Drug Advisory Service,

VERNON HOCHULI Kent ME7 4LP

\section{Does atenolol have an effect on calcium metabolism?}

SIR,-Dr C J Bushe (23 May, p 1324) reports the hypocalcaemic effect of the selective $\beta$ blocker atenolol and suggests that this may be due to blockade of adrenergic mediated release of parathyroid hormone. This is not, however, the whole story as experimental in vitro evidence shows that the non-selective $\beta$ blocker propranolol may inhibit bone resorption stimulated by parathyroid hormone, vitamin D metabolites, prostaglandins, and osteoclast activating factor.' ${ }^{\prime}$ Furthermore, propranolol can inhibit thyroid hormone stimulated bone resorption in vitro as effectively as salmon calcitonin $^{2}$ and can lower serum calcium concentration in the thyrotoxic patient. ${ }^{3}$ The thyroid hormones directly stimulate cortical osteoclast activity, increasing both bone turnover and the serum calcium concentration while secondarily decreasing the serum parathyroid hormone concentration, often to non-detectable levels. Propranolol probably acts by direct inhibition of osteoclast activity, and inhibition may be more effective when bone turnover is increased. ${ }^{4}$ This site of action would explain the hypocalcaemic effect of atenolol seen in primary hyperparathyroidism and sarcoidosis, and alterations in the plasma and urinary phosphate and 24 hour urinary calcium concentrations would reflect the reduction in bone turnover and a secondary response of the parathyroid glands.

Though $\beta$ adrenergic blockade may reduce secretion of parathyroid hormone, measurement temporally of serum parathyroid hormone concentration, urinary renal cyclic adenosine monophosphate concentration, and hydroxyproline secretion in response to atenolol in these patients would probably confirm a direct site of bone action that was independent of parathyroid hormone.

Nuffield Department of Surgery,

ROBERT M JONES

John Radcliffe Hospital,

Oxford

1 Hermann-Erlee MPM, Meer JM. The effects of dibutyryl cyclic AMP, aminophylline and propranolol on PTE-induced bone reabsorption in vitro. Endocrinology 1974;94:424-34.

2 Mundy GR, Shapiro JL, Bandelin JG, et al. Direct stimulation of bone reabsorption by thyroid hormones. $f$ Clin Invest 1976;58: 529-34.
3 Rude RK, Oldham SB, Singer FR, Nicoloff JT. Treatment of thyrotoxic hypercal

4 Jones RM, Davidson CM. Thyrotoxicosis and the hungry bone syndrome. f R Coll Surg Edinb 1987;32:24-8.

SIR,-One observation is interesting, two are publishable seems to be the basis of Dr C J Bushe's report (23 May, p 1324), and we therefore report a case that is contrary to his experience.

A 41 year old woman with hypercalcaemia due to primary hyperparathyroidism had been treated with atenolol $100 \mathrm{mg}$ daily for hypertension. This was stopped before entry to a double blind study comparing the hypotensive effect of atenolol, placebo, and an angiotensin converting enzyme inhibitor. The table shows her plasma calcium, phosphate, and albumin concentrations on four occasions, twice while taking atenolol (in different doses), once when receiving no treatment (for five days), and once while receiving placebo. All treatments had been given for at least three weeks.

Patient's calcium, phosphate, and albumin concentrations while receiving atenolol or no treatment

\begin{tabular}{|c|c|c|c|c|c|}
\hline & $\begin{array}{l}\text { Normal } \\
\text { range }\end{array}$ & $\begin{array}{l}\text { Atenolol } \\
100 \mathrm{mg}\end{array}$ & $\begin{array}{c}\text { No } \\
\text { treatment }\end{array}$ & $\begin{array}{c}\text { Atenolol } \\
50 \mathrm{mg}\end{array}$ & Placebo \\
\hline $\begin{array}{l}\text { Total calcium } \\
\text { (mmol//) } \\
\text { Phosphate }\end{array}$ & $2 \cdot 12-2 \cdot 62$ & 2.69 & $2 \cdot 82$ & 3.00 . & 2.89 \\
\hline $\begin{array}{c}(\mathrm{mmol} / \mathrm{l}) \\
\text { Albumin }(\mathrm{g} / \mathrm{l})\end{array}$ & $\begin{array}{c}0 \cdot 8-1 \cdot 4 \\
36-47\end{array}$ & $\begin{array}{l}0.75 \\
38\end{array}$ & $\begin{array}{l}0.44 \\
41\end{array}$ & $4^{1 \cdot 08}$ & $\begin{array}{l}0.73 \\
41\end{array}$ \\
\hline
\end{tabular}

This case illustrates the wide variation in plasma calcium and phosphate concentrations possible in patients with this condition. Both the highest and lowest plasma calcium concentrations occurred during atenolol treatment, and the phosphate concentrations were similar while the patient was taking atenolol $100 \mathrm{mg}$ daily and placebo. Dr Bushe did not state whether the blood samples were taken under standard conditions (fasting, no venous stasis). His observations might be explained by random variation, and we need much better evidence before accepting that atenolol has any effect on calcium metabolism.

S FREESTONE T M MACDONALD University Department of Clinical Pharmacology,

Royal Infirmary,

Edinburgh EH3 9XW

\section{The future role of midwives}

SIR,-Professor Peter W Howie (13 June, p 1502) highlights the modern dilemmas of maternity care. Without being too simplistic, two basic and contrasting care philosophies may be identified from the existing maternity medical services. The general practitioner obstetrician perceives that the safety of his care results from the relationship he creates with his patients. This is now usually limited to shared antenatal and postnatal care, but evidence shows that when carried through to intrapartum care it leads to the non-interventionist approach much favoured by midwives and many rospective mothers.

The hospital obstetrician by the nature of his working curriculum cannot achieve the same continuity of relationship with his patients, and so the safety of his care depends on the accuracy of transferable records and a strict adherence to protocols and procedures that cover every eventuality. This has led to criticism that hospital obstetricians do not respond to individual patients' needs and undertake too much medical intervention in normal labour, though this is defended by the increasing overall safety of childbirth

Midwives fall between these two philosophies. Their traditional role is that of the non-interventionist carer, but their salaried 37 and a half hour week pushes them towards the specialist camp. The concept of the midwife team working independently of other health care professionals is, as Professor Howie points out, unrealistic, but the dilemma might be overcome by more midwives having closer identification with primary health care teams. Consideration might be given to the idea of combining midwives' contracts with their district health authorities and the family practitioner services using a shared salaried structure. If the general practitioner ancillary payment scheme was used this part of the salary could be financed by more shared antenatal care and its conversion to more full general practitioner care, with the midwife using her skills appropriately.

Such a concept might carry the seeds of a job structure that would give our midwives more professional satisfaction and reduce the almost universally expressed feeling of being undervalued. It would, of course, lead to general practitioners participating more in childbirth, but as most vocational training schemes now include an obstetric senior house officer post it is not unreasonable to expect general practitioners to be able to oversee the normal and refer the complicated.

Paxton Green Health Centre,

M P ROSEVEARE London SE21 8AU

\section{Pinch skin grafting or porcine dermis in} venous ulcers

SIR,-At least three of the comments on my letter (23 May, p 1352) by Messrs K R Poskitt and Charles McCollum are not supported by the work of others.

Firstly, they seem to base their concepts on the hypothesis of Professor Browse that pericapillary fibrin forms a diffusion barrier to oxygen, resulting in tissue hypoxia, and on this basis they propose two different forms of treatment-namely, compression bandaging $(40 \mathrm{~mm} \mathrm{Hg}$ ) for one week and, in the case of large ulcers, pinch grafting. They did not explain how the treatments overcame the hypoxia resulting from the fibrin cuff, nor is it easy to see how the fibrin cuff could have been affected by the treatments. Dodd et al, however, showed that transcutaneous oxygen tensions in the skin of the foot were higher in patients with ulcers than in controls when the subjects were in the recumbent position, but much lower when subjects were standing. ${ }^{1}$ It is unlikely that vascular changes occurred when the body changed from the horizontal to the vertical plane.

Therefore it seems reasonable to suggest that the fall in oxygen pressure results from a reduction in the rate of blood flow, which would result in haemorrheological changes. Reduction in the rate of blood flow results in thixotropic amplification of blood viscosity, which adds to the resistance to flow. Evidence suggests that hypoxia reduces red cell deformability, and this could impair flow in the smallest capillaries. As Dodd et al pointed out, their measurements of oxygen pressures in the skin of the foot disproved the existence of a fibrin related oxygen barrier and provided a basis for understanding why bed rest is effective in the management of venous ulcers.'

Secondly, Messrs Poskett and McCallum proposed that pinch grafting increased the rate of healing in large ulcers. According to Monk and Sarkany, two thirds of grafted ulcers recurred 